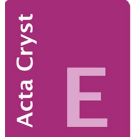
COMMUNICATIONS

ISSN 2056-9890

\section{Crystal structure of 2-methyl-N-\{[2- (pyridin-2-yl)ethyl]carbamothioyl\}- benzamide}

\author{
Nadiah Ameram and Farook Adam* \\ School of Chemical Sciences, Universiti Sains Malaysia, 11800 Georgetown,
} Penang, Malaysia. *Correspondence e-mail: farookdr@gmail.com

Received 12 July 2015; accepted 14 July 2015

Edited by H. Stoeckli-Evans, University of Neuchâtel, Switzerland

In the title compound, $\mathrm{C}_{16} \mathrm{H}_{17} \mathrm{~N}_{3} \mathrm{OS}$, a benzoyl thiourea derivative, the planes of the pyridine and benzene rings are inclined to one another by $66.54(9)^{\circ}$. There is an intramolecular $\mathrm{N}-\mathrm{H} \cdots \mathrm{O}$ hydrogen bond present forming an $S(6)$ ring motif. In the crystal, molecules are linked via pairs of $\mathrm{N}-$ $\mathrm{H} \cdots \mathrm{N}$ hydrogen bonds, forming inversion dimers, which are reinforced by pairs of $\mathrm{C}-\mathrm{H} \cdots \mathrm{S}$ hydrogen bonds. The dimers are linked via $\mathrm{C}-\mathrm{H} \cdots \pi$ interactions, forming ribbons along [010].

Keywords: crystal structure; benzamide; carbonyl thiourea; inversion dimers; hydrogen bonding.

CCDC reference: 1412857

\section{Related literature}

For the crystal structure of the 4-methyl derivative, 4-methyl$N$-\{[2-(pyridin-2-yl)ethyl]carbamothioyl\}benzamide, see: Adam et al. (2014). For the crystal structure of $N$-carbamothioyl-2methylbenzamide, see: Adam et al. (2015).<smiles>Cc1ccccc1C(=O)NC(=S)NCCc1ccccn1</smiles>

\section{Experimental}

\subsection{Crystal data}

$\mathrm{C}_{16} \mathrm{H}_{17} \mathrm{~N}_{3} \mathrm{OS}$

$M_{r}=299.38$

Triclinic, $P \overline{1}$

$a=8.5434(4) \AA$ $b=8.7477(4) \AA$

$c=11.0530(5) \AA$

$\alpha=86.1868(13)^{\circ}$

$\beta=83.3739(13)^{\circ}$

$\gamma=73.8746(13)^{\circ}$

$V=787.73(6) \AA^{3}$

\subsection{Data collection}

Bruker APEX DUO CCD areadetector diffractometer

Absorption correction: multi-scan

(SADABS; Bruker, 2009)

$T_{\min }=0.670, T_{\max }=0.867$

\subsection{Refinement}

$R\left[F^{2}>2 \sigma\left(F^{2}\right)\right]=0.045$

$w R\left(F^{2}\right)=0.129$

$S=1.03$

4698 reflections

199 parameters

Table 1

Hydrogen-bond geometry $\left(\AA{ }^{\circ}\right)$.

$\mathrm{Cg} 2$ is the centroid of the $\mathrm{C} 1-\mathrm{C} 6$ ring.

\begin{tabular}{lllll}
\hline$D-\mathrm{H} \cdots A$ & $D-\mathrm{H}$ & $\mathrm{H} \cdots A$ & $D \cdots A$ & $D-\mathrm{H} \cdots A$ \\
\hline $\mathrm{N} 2-\mathrm{H} 1 N 2 \cdots \mathrm{O} 1$ & $0.842(17)$ & $1.971(18)$ & $2.6550(16)$ & $137.8(16)$ \\
$\mathrm{N} 1-\mathrm{H} 1 N 1 \cdots \mathrm{N} 3^{\mathrm{i}}$ & $0.875(17)$ & $2.061(17)$ & $2.9366(17)$ & $180(3)$ \\
$\mathrm{C} 16-\mathrm{H} 16 A \cdots \mathrm{S} 1^{\mathrm{i}}$ & 0.96 & 2.86 & $3.782(2)$ & 162 \\
$\mathrm{C} 12-\mathrm{H} 12 A \cdots \mathrm{Cg} 2^{\mathrm{ii}}$ & 0.93 & 2.92 & $3.7724(19)$ & 153 \\
\hline
\end{tabular}

Symmetry codes: (i) $-x+1,-y+2,-z$; (ii) $-x+1,-y+1,-z$.

Data collection: APEX2 (Bruker, 2009); cell refinement: SAINT (Bruker, 2009); data reduction: $S A I N T$; program(s) used to solve structure: SHELXS2013 (Sheldrick, 2008); program(s) used to refine structure: SHELXL2014 (Sheldrick, 2015); molecular graphics: SHELXTL (Sheldrick, 2008) and Mercury (Macrae et al., 2008); software used to prepare material for publication: SHELXL2014 and PLATON (Spek, 2009).

\section{Acknowledgements}

The authors thank the Universiti Sains Malaysia for research grant Nos. PKIMIA846017 and RU-1001/PKIMIA/811269, which partially supported this work.

Supporting information for this paper is available from the IUCr electronic archives (Reference: SU5173).

\section{References}

Adam, F., Ameram, N. \& Eltayeb, N. E. (2014). Acta Cryst. E70, o885.

Adam, F., Ameram, N. \& Tan, W. M. (2015). Acta Cryst. E71, o425.

Bruker (2009). APEX2, SAINT and SADABS. Bruker AXS Inc., Madison, Wisconsin, USA.

Macrae, C. F., Bruno, I. J., Chisholm, J. A., Edgington, P. R., McCabe, P., Pidcock, E., Rodriguez-Monge, L., Taylor, R., van de Streek, J. \& Wood, P. A. (2008). J. Appl. Cryst. 41, 466-470.

Sheldrick, G. M. (2008). Acta Cryst. A64, 112-122.

Sheldrick, G. M. (2015). Acta Cryst. C71, 3-8.

Spek, A. L. (2009). Acta Cryst. D65, 148-155. 


\section{supporting information}

Acta Cryst. (2015). E71, o636 [https://doi.org/10.1107/S2056989015013559]

\section{Crystal structure of 2-methyl- $N$-\{[2-(pyridin-2-yl)ethyl] carbamo-}

\section{thioyl\}benzamide}

\section{Nadiah Ameram and Farook Adam}

\section{S1. Synthesis and crystallization}

Ortho Benzoyl chloride $(13 \mathrm{mmol})$ was added drop wise to a stirred acetone solution $(30 \mathrm{ml})$ of ammonium thiocyanate (13 mmol). Stirring was continued for $10 \mathrm{~min}$. A solution of ethyl pyridine in acetone was then added and the reaction mixture was refluxed for $3 \mathrm{~h}$, after which the solution was poured into a beaker containing some ice cubes. The resulting precipitate was collected by titration, washed several times with a cold ethanol/water mixture and purified by recrystallization from an ethanol solution, yielding colourless plate-like crystals.

\section{S1.1. Refinement}

Crystal data, data collection and structure refinement details are summarized in Table 2. The NH H-atoms were located in a difference Fourier map and freely refined. The $\mathrm{C}$-bound $\mathrm{H}$ atoms were positioned geometrically and refined using a riding model: $\mathrm{C}-\mathrm{H}=0.93-0.96 \AA$ with $\mathrm{U}_{\mathrm{iso}}(\mathrm{H})=1.5 \mathrm{U}_{\mathrm{eq}}(\mathrm{C})$ for methyl $\mathrm{H}$ atoms and $1.2 \mathrm{U}_{\mathrm{eq}}(\mathrm{C})$ for other $\mathrm{H}$ atoms.

\section{S2. Structural commentary}

The title compound, Fig. 1, is a benzoyl thiourea derivative with the pyridine and benzene rings being inclined to one another by 66.54 (9) ${ }^{\circ}$. The bond lengths and angles are very similar to those observed for the analogous structure, 4methyl-N-[2-(pyridin-2-yl)ethylcarbamothioyl]benzamide (Adam et al., 2014). However, in the 4-methyl derivative the pyridine and benzene rings are inclined to one another by $71.33(15)^{\circ}$. There is an intramolecular $\mathrm{N}-\mathrm{H} \cdots \mathrm{O}$ hydrogen bond present in both molecules forming an S(6) ring motif (for the title compound, see Table 1 and Fig. 1).

In the crystal, molecules are linked via pairs of $\mathrm{N}-\mathrm{H} \cdots \mathrm{N}$ hydrogen bonds forming inversion dimers which are reinforced by pairs of $\mathrm{C}-\mathrm{H} \cdots \mathrm{S}$ hydrogen bonds (Fig. 2 and Table 1). The dimers are linked via $\mathrm{C}-\mathrm{H} \cdots \pi$ interactions forming ribbons along [010]; Table 1 and Fig. 2. 


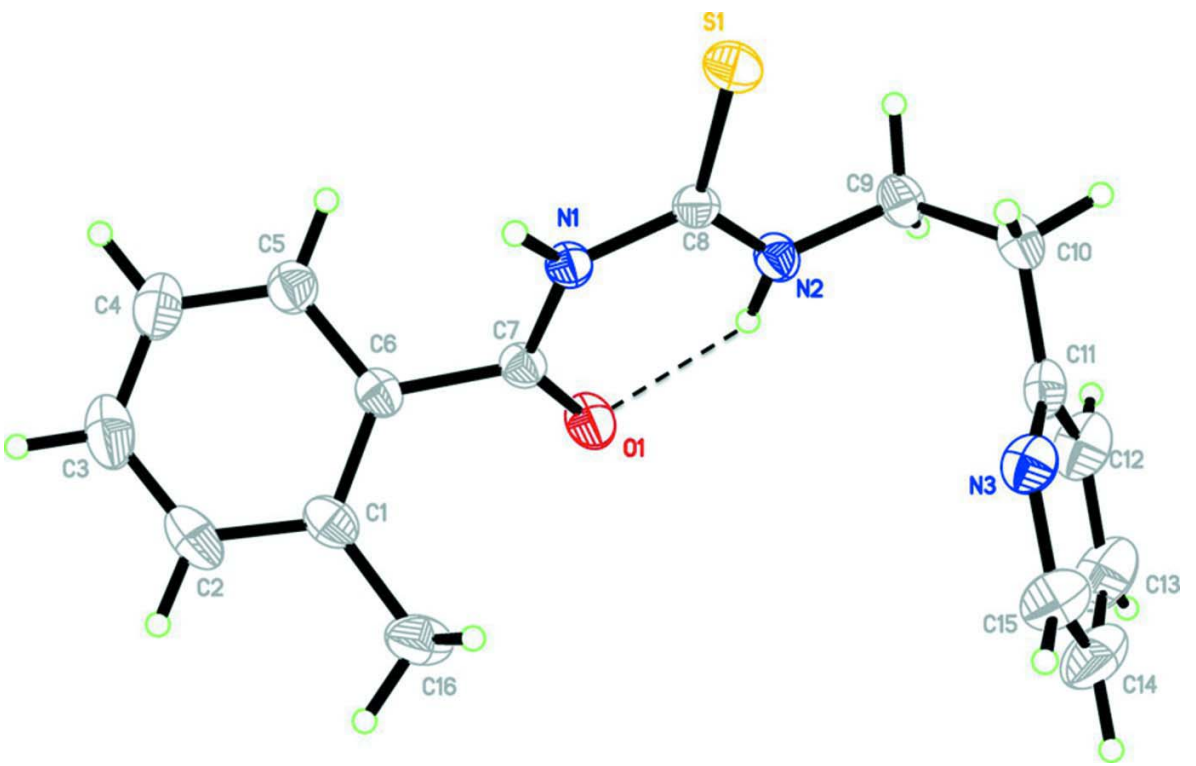

Figure 1

The molecular structure of the title compound, with atom labelling. Displacement ellipsoids are drawn at the 50\% probability level. The intramolecular $\mathrm{N}-\mathrm{H} \cdots \mathrm{O}$ hydrogen bond is shown as a dashed line (see Table 1 for details).

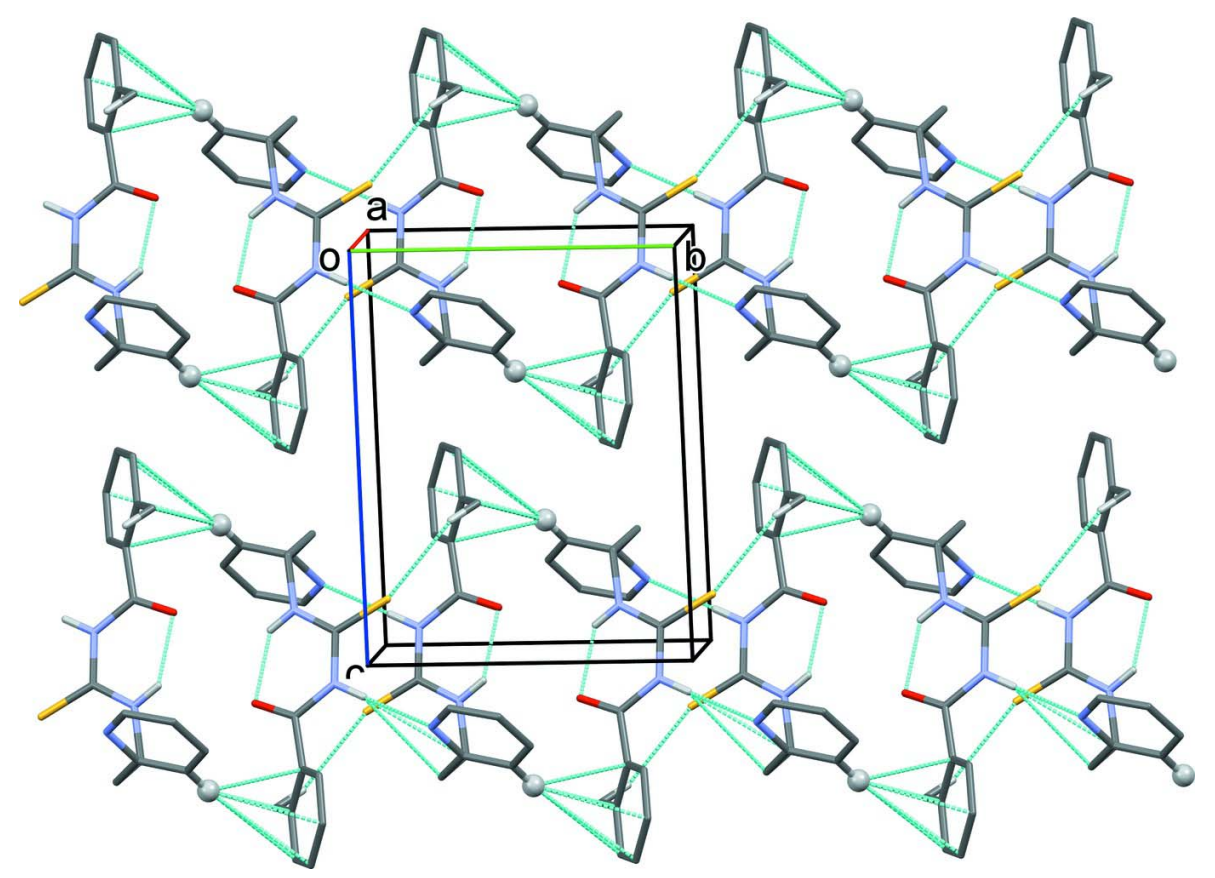

Figure 2

A view along the $a$ axis of the crystal packing of the title compound. The hydrogen bonds and C-H $\cdots \pi$ interactions (H atom a grey ball) are shown as dashed lines (see Table 1 for details). 
2-Methyl-N-\{[2-(pyridin-2-yl)ethyl] carbamothioyl\}benzamide

Crystal data

$\mathrm{C}_{16} \mathrm{H}_{17} \mathrm{~N}_{3} \mathrm{OS}$

$M_{r}=299.38$

Triclinic, $P \overline{1}$

$a=8.5434$ (4) $\AA$

$b=8.7477(4) \AA$

$c=11.0530(5) \AA$

$\alpha=86.1868(13)^{\circ}$

$\beta=83.3739(13)^{\circ}$

$\gamma=73.8746(13)^{\circ}$

$V=787.73(6) \AA^{3}$

\section{Data collection}

Bruker APEX DUO CCD area-detector diffractometer

Radiation source: fine-focus sealed tube

Graphite monochromator

$\varphi$ and $\omega$ scans

Absorption correction: multi-scan

(SADABS; Bruker, 2009)

$T_{\min }=0.670, T_{\max }=0.867$

\section{Refinement}

Refinement on $F^{2}$

Least-squares matrix: full

$R\left[F^{2}>2 \sigma\left(F^{2}\right)\right]=0.045$

$w R\left(F^{2}\right)=0.129$

$S=1.03$

4698 reflections

199 parameters

0 restraints

Primary atom site location: structure-invariant direct methods
$Z=2$

$F(000)=316$

$D_{\mathrm{x}}=1.262 \mathrm{Mg} \mathrm{m}^{-3}$

Mo $K \alpha$ radiation, $\lambda=0.71073 \AA$

Cell parameters from 8347 reflections

$\theta=2.4-28.3^{\circ}$

$\mu=0.21 \mathrm{~mm}^{-1}$

$T=100 \mathrm{~K}$

Plate, colourless

$0.53 \times 0.42 \times 0.22 \mathrm{~mm}$

30520 measured reflections

4698 independent reflections

3412 reflections with $I>2 \sigma(I)$

$R_{\text {int }}=0.045$

$\theta_{\text {max }}=30.3^{\circ}, \theta_{\min }=1.9^{\circ}$

$h=-12 \rightarrow 12$

$k=-12 \rightarrow 12$

$l=-15 \rightarrow 15$

Secondary atom site location: difference Fourier map

Hydrogen site location: mixed

$\mathrm{H}$ atoms treated by a mixture of independent and constrained refinement

$w=1 /\left[\sigma^{2}\left(F_{\mathrm{o}}^{2}\right)+(0.0515 P)^{2}+0.2342 P\right]$

where $P=\left(F_{\mathrm{o}}^{2}+2 F_{\mathrm{c}}^{2}\right) / 3$

$(\Delta / \sigma)_{\max }<0.001$

$\Delta \rho_{\max }=0.37 \mathrm{e} \AA^{-3}$

$\Delta \rho_{\min }=-0.22$ e $\AA^{-3}$

Special details

Geometry. All e.s.d.'s (except the e.s.d. in the dihedral angle between two 1.s. planes) are estimated using the full covariance matrix. The cell e.s.d.'s are taken into account individually in the estimation of e.s.d.'s in distances, angles and torsion angles; correlations between e.s.d.'s in cell parameters are only used when they are defined by crystal symmetry. An approximate (isotropic) treatment of cell e.s.d.'s is used for estimating e.s.d.'s involving 1.s. planes.

Fractional atomic coordinates and isotropic or equivalent isotropic displacement parameters $\left(\AA^{2}\right)$

\begin{tabular}{lllll}
\hline & $x$ & $y$ & $z$ & $U_{\text {iso }} * / U_{\text {eq }}$ \\
\hline S1 & $0.29858(5)$ & $1.05179(5)$ & $-0.14484(4)$ & $0.05473(14)$ \\
O1 & $0.46415(15)$ & $0.62159(12)$ & $0.10945(10)$ & $0.0552(3)$ \\
N1 & $0.32417(14)$ & $0.87622(13)$ & $0.05803(10)$ & $0.0367(2)$ \\
N2 & $0.47718(15)$ & $0.75403(15)$ & $-0.11337(11)$ & $0.0408(3)$ \\
N3 & $0.87338(16)$ & $0.81820(15)$ & $-0.15598(13)$ & $0.0499(3)$ \\
C1 & $0.3515(2)$ & $0.72438(18)$ & $0.36636(14)$ & $0.0482(3)$ \\
C2 & $0.2522(3)$ & $0.7379(2)$ & $0.47681(15)$ & $0.0654(5)$ \\
H2A & 0.3012 & 0.7083 & 0.5488 & $0.079 *$
\end{tabular}




$\begin{array}{lllll}\text { C3 } & 0.0847(3) & 0.7934(3) & 0.48282(18) & 0.0731(6) \\ \text { H3A } & 0.0226 & 0.7999 & 0.5581 & 0.088^{*} \\ \text { C4 } & 0.0088(2) & 0.8393(2) & 0.37890(18) & 0.0638(5) \\ \text { H4A } & -0.1046 & 0.8769 & 0.3828 & 0.077^{*} \\ \text { C5 } & 0.10295(19) & 0.82906(18) & 0.26820(15) & 0.0478(3) \\ \text { H5A } & 0.0522 & 0.8619 & 0.1972 & 0.057^{*} \\ \text { C6 } & 0.27224(18) & 0.77065(15) & 0.26075(12) & 0.0392(3) \\ \text { C7 } & 0.36489(17) & 0.74777(16) & 0.13728(12) & 0.0384(3) \\ \text { C8 } & 0.37507(16) & 0.88350(16) & -0.06595(12) & 0.0359(3) \\ \text { C9 } & 0.5275(2) & 0.73791(19) & -0.24280(13) & 0.0462(3) \\ \text { H9A } & 0.4348 & 0.7906 & -0.2875 & 0.055^{*} \\ \text { H9B } & 0.5589 & 0.6259 & -0.2610 & 0.055^{*} \\ \text { C10 } & 0.6698(2) & 0.80764(19) & -0.28709(14) & 0.0487(3) \\ \text { H10A } & 0.6926 & 0.7974 & -0.3746 & 0.058^{*} \\ \text { H10B } & 0.6371 & 0.9203 & -0.2709 & 0.058^{*} \\ \text { C11 } & 0.82339(18) & 0.73009(17) & -0.22889(14) & 0.0445(3) \\ \text { C12 } & 0.9112(2) & 0.5738(2) & -0.2508(2) & 0.0661(5) \\ \text { H12A } & 0.8745 & 0.5138 & -0.3019 & 0.079^{*} \\ \text { C13 } & 1.0517(3) & 0.5084(2) & -0.1970(3) & 0.0805(7) \\ \text { H13A } & 1.1105 & 0.4032 & -0.2102 & 0.097^{*} \\ \text { C14 } & 1.1056(2) & 0.5992(3) & -0.1232(2) & 0.0779(6) \\ \text { H14A } & 1.2021 & 0.5582 & -0.0867 & 0.093^{*} \\ \text { C15 } & 1.0123(2) & 0.7523(2) & -0.1053(2) & 0.0676(5) \\ \text { H15A } & 1.0477 & 0.8142 & -0.0549 & 0.081^{*} \\ \text { C16 } & 0.5335(2) & 0.6686(3) & 0.36477(18) & 0.0661(5) \\ \text { H16A } & 0.5808 & 0.7494 & 0.3264 & 0.099^{*} \\ \text { H16B } & 0.5743 & 0.5728 & 0.3200 & 0.099^{*} \\ \text { H16C } & 0.5624 & 0.6477 & 0.4468 & 0.099^{*} \\ \text { H1N2 } & 0.508(2) & 0.676(2) & -0.0647(16) & 0.049(5)^{*} \\ \text { H1N1 } & 0.265(2) & 0.967(2) & 0.0876(16) & 0.049(5)^{*}\end{array}$

Atomic displacement parameters $\left(\AA^{2}\right)$

\begin{tabular}{lllllll}
\hline & $U^{11}$ & $U^{22}$ & $U^{33}$ & $U^{12}$ & $U^{13}$ & $U^{23}$ \\
\hline S1 & $0.0565(2)$ & $0.0474(2)$ & $0.0519(2)$ & $-0.00366(17)$ & $-0.00764(18)$ & $0.01618(17)$ \\
O1 & $0.0692(7)$ & $0.0369(5)$ & $0.0446(6)$ & $0.0061(5)$ & $0.0018(5)$ & $0.0038(4)$ \\
N1 & $0.0395(6)$ & $0.0309(5)$ & $0.0360(6)$ & $-0.0043(4)$ & $-0.0032(4)$ & $0.0010(4)$ \\
N2 & $0.0463(6)$ & $0.0384(6)$ & $0.0355(6)$ & $-0.0096(5)$ & $-0.0016(5)$ & $0.0026(5)$ \\
N3 & $0.0483(7)$ & $0.0395(6)$ & $0.0586(8)$ & $-0.0079(5)$ & $-0.0005(6)$ & $-0.0052(6)$ \\
C1 & $0.0641(9)$ & $0.0415(7)$ & $0.0405(7)$ & $-0.0166(7)$ & $-0.0103(7)$ & $0.0054(6)$ \\
C2 & $0.0994(15)$ & $0.0627(11)$ & $0.0355(8)$ & $-0.0253(10)$ & $-0.0080(9)$ & $0.0049(7)$ \\
C3 & $0.0886(15)$ & $0.0695(12)$ & $0.0523(10)$ & $-0.0179(11)$ & $0.0201(10)$ & $-0.0027(9)$ \\
C4 & $0.0601(10)$ & $0.0592(10)$ & $0.0635(11)$ & $-0.0100(8)$ & $0.0140(9)$ & $-0.0032(8)$ \\
C5 & $0.0506(8)$ & $0.0436(8)$ & $0.0458(8)$ & $-0.0086(6)$ & $-0.0015(6)$ & $-0.0008(6)$ \\
C6 & $0.0499(8)$ & $0.0310(6)$ & $0.0357(7)$ & $-0.0099(5)$ & $-0.0038(6)$ & $0.0006(5)$ \\
C7 & $0.0430(7)$ & $0.0331(6)$ & $0.0368(7)$ & $-0.0067(5)$ & $-0.0054(5)$ & $0.0006(5)$ \\
C8 & $0.0345(6)$ & $0.0375(6)$ & $0.0369(6)$ & $-0.0111(5)$ & $-0.0072(5)$ & $0.0028(5)$ \\
C9 & $0.0559(9)$ & $0.0488(8)$ & $0.0360(7)$ & $-0.0176(7)$ & $-0.0025(6)$ & $-0.0056(6)$
\end{tabular}




\begin{tabular}{lllllll}
\hline & & & & \\
C10 & $0.0598(9)$ & $0.0483(8)$ & $0.0374(7)$ & $-0.0171(7)$ & $0.0017(6)$ & $0.0012(6)$ \\
C11 & $0.0474(8)$ & $0.0380(7)$ & $0.0449(8)$ & $-0.0131(6)$ & $0.0112(6)$ & $-0.0009(6)$ \\
C12 & $0.0633(11)$ & $0.0428(9)$ & $0.0883(14)$ & $-0.0116(8)$ & $0.0089(10)$ & $-0.0158(9)$ \\
C13 & $0.0581(11)$ & $0.0424(9)$ & $0.128(2)$ & $0.0000(8)$ & $0.0105(12)$ & $-0.0077(11)$ \\
C14 & $0.0437(9)$ & $0.0661(12)$ & $0.1145(18)$ & $-0.0032(9)$ & $-0.0051(10)$ & $0.0084(12)$ \\
C15 & $0.0534(10)$ & $0.0612(11)$ & $0.0871(14)$ & $-0.0109(8)$ & $-0.0126(9)$ & $-0.0062(10)$ \\
C16 & $0.0702(12)$ & $0.0708(12)$ & $0.0607(11)$ & $-0.0217(9)$ & $-0.0261(9)$ & $0.0174(9)$ \\
\hline
\end{tabular}

Geometric parameters $\left(\AA,{ }^{\circ}\right)$

\begin{tabular}{|c|c|c|c|}
\hline $\mathrm{S} 1-\mathrm{C} 8$ & $1.6675(13)$ & $\mathrm{C} 5-\mathrm{H} 5 \mathrm{~A}$ & 0.9300 \\
\hline $\mathrm{O} 1-\mathrm{C} 7$ & $1.2236(16)$ & $\mathrm{C} 6-\mathrm{C} 7$ & $1.4926(19)$ \\
\hline $\mathrm{N} 1-\mathrm{C} 7$ & $1.3686(17)$ & $\mathrm{C} 9-\mathrm{C} 10$ & $1.525(2)$ \\
\hline $\mathrm{N} 1-\mathrm{C} 8$ & $1.3919(17)$ & C9-H9A & 0.9700 \\
\hline $\mathrm{N} 1-\mathrm{H} 1 \mathrm{~N} 1$ & $0.874(18)$ & C9-H9B & 0.9700 \\
\hline $\mathrm{N} 2-\mathrm{C} 8$ & $1.3203(18)$ & $\mathrm{C} 10-\mathrm{C} 11$ & $1.496(2)$ \\
\hline $\mathrm{N} 2-\mathrm{C} 9$ & $1.4499(18)$ & $\mathrm{C} 10-\mathrm{H} 10 \mathrm{~A}$ & 0.9700 \\
\hline $\mathrm{N} 2-\mathrm{H} 1 \mathrm{~N} 2$ & $0.841(19)$ & $\mathrm{C} 10-\mathrm{H} 10 \mathrm{~B}$ & 0.9700 \\
\hline N3-C11 & $1.331(2)$ & $\mathrm{C} 11-\mathrm{C} 12$ & $1.387(2)$ \\
\hline N3-C15 & $1.336(2)$ & $\mathrm{C} 12-\mathrm{C} 13$ & $1.364(3)$ \\
\hline $\mathrm{C} 1-\mathrm{C} 2$ & $1.396(2)$ & $\mathrm{C} 12-\mathrm{H} 12 \mathrm{~A}$ & 0.9300 \\
\hline $\mathrm{C} 1-\mathrm{C} 6$ & $1.397(2)$ & $\mathrm{C} 13-\mathrm{C} 14$ & $1.372(3)$ \\
\hline $\mathrm{C} 1-\mathrm{C} 16$ & $1.494(3)$ & $\mathrm{C} 13-\mathrm{H} 13 \mathrm{~A}$ & 0.9300 \\
\hline $\mathrm{C} 2-\mathrm{C} 3$ & $1.372(3)$ & $\mathrm{C} 14-\mathrm{C} 15$ & $1.369(3)$ \\
\hline $\mathrm{C} 2-\mathrm{H} 2 \mathrm{~A}$ & 0.9300 & $\mathrm{C} 14-\mathrm{H} 14 \mathrm{~A}$ & 0.9300 \\
\hline $\mathrm{C} 3-\mathrm{C} 4$ & $1.366(3)$ & $\mathrm{C} 15-\mathrm{H} 15 \mathrm{~A}$ & 0.9300 \\
\hline $\mathrm{C} 3-\mathrm{H} 3 \mathrm{~A}$ & 0.9300 & C16-H16A & 0.9600 \\
\hline $\mathrm{C} 4-\mathrm{C} 5$ & $1.378(2)$ & C16-H16B & 0.9600 \\
\hline $\mathrm{C} 4-\mathrm{H} 4 \mathrm{~A}$ & 0.9300 & $\mathrm{C} 16-\mathrm{H} 16 \mathrm{C}$ & 0.9600 \\
\hline $\mathrm{C} 5-\mathrm{C} 6$ & $1.388(2)$ & & \\
\hline $\mathrm{C} 7-\mathrm{N} 1-\mathrm{C} 8$ & $127.33(12)$ & $\mathrm{N} 2-\mathrm{C} 9-\mathrm{H} 9 \mathrm{~A}$ & 108.9 \\
\hline $\mathrm{C} 7-\mathrm{N} 1-\mathrm{H} 1 \mathrm{~N} 1$ & $118.0(12)$ & $\mathrm{C} 10-\mathrm{C} 9-\mathrm{H} 9 \mathrm{~A}$ & 108.9 \\
\hline $\mathrm{C} 8-\mathrm{N} 1-\mathrm{H} 1 \mathrm{~N} 1$ & $114.5(12)$ & $\mathrm{N} 2-\mathrm{C} 9-\mathrm{H} 9 \mathrm{~B}$ & 108.9 \\
\hline $\mathrm{C} 8-\mathrm{N} 2-\mathrm{C} 9$ & $123.54(13)$ & $\mathrm{C} 10-\mathrm{C} 9-\mathrm{H} 9 \mathrm{~B}$ & 108.9 \\
\hline $\mathrm{C} 8-\mathrm{N} 2-\mathrm{H} 1 \mathrm{~N} 2$ & $116.3(12)$ & H9A-C9-H9B & 107.7 \\
\hline $\mathrm{C} 9-\mathrm{N} 2-\mathrm{H} 1 \mathrm{~N} 2$ & $120.0(12)$ & $\mathrm{C} 11-\mathrm{C} 10-\mathrm{C} 9$ & $113.91(13)$ \\
\hline $\mathrm{C} 11-\mathrm{N} 3-\mathrm{C} 15$ & $118.03(15)$ & $\mathrm{C} 11-\mathrm{C} 10-\mathrm{H} 10 \mathrm{~A}$ & 108.8 \\
\hline $\mathrm{C} 2-\mathrm{C} 1-\mathrm{C} 6$ & $116.77(16)$ & $\mathrm{C} 9-\mathrm{C} 10-\mathrm{H} 10 \mathrm{~A}$ & 108.8 \\
\hline $\mathrm{C} 2-\mathrm{C} 1-\mathrm{C} 16$ & $120.27(16)$ & $\mathrm{C} 11-\mathrm{C} 10-\mathrm{H} 10 \mathrm{~B}$ & 108.8 \\
\hline $\mathrm{C} 6-\mathrm{C} 1-\mathrm{C} 16$ & $122.91(15)$ & $\mathrm{C} 9-\mathrm{C} 10-\mathrm{H} 10 \mathrm{~B}$ & 108.8 \\
\hline $\mathrm{C} 3-\mathrm{C} 2-\mathrm{C} 1$ & $122.25(17)$ & $\mathrm{H} 10 \mathrm{~A}-\mathrm{C} 10-\mathrm{H} 10 \mathrm{~B}$ & 107.7 \\
\hline $\mathrm{C} 3-\mathrm{C} 2-\mathrm{H} 2 \mathrm{~A}$ & 118.9 & $\mathrm{~N} 3-\mathrm{C} 11-\mathrm{C} 12$ & $121.26(16)$ \\
\hline $\mathrm{C} 1-\mathrm{C} 2-\mathrm{H} 2 \mathrm{~A}$ & 118.9 & $\mathrm{~N} 3-\mathrm{C} 11-\mathrm{C} 10$ & $117.10(13)$ \\
\hline $\mathrm{C} 4-\mathrm{C} 3-\mathrm{C} 2$ & $120.40(17)$ & $\mathrm{C} 12-\mathrm{C} 11-\mathrm{C} 10$ & $121.64(16)$ \\
\hline $\mathrm{C} 4-\mathrm{C} 3-\mathrm{H} 3 \mathrm{~A}$ & 119.8 & $\mathrm{C} 13-\mathrm{C} 12-\mathrm{C} 11$ & $119.68(19)$ \\
\hline $\mathrm{C} 2-\mathrm{C} 3-\mathrm{H} 3 \mathrm{~A}$ & 119.8 & $\mathrm{C} 13-\mathrm{C} 12-\mathrm{H} 12 \mathrm{~A}$ & 120.2 \\
\hline $\mathrm{C} 3-\mathrm{C} 4-\mathrm{C} 5$ & $118.99(18)$ & $\mathrm{C} 11-\mathrm{C} 12-\mathrm{H} 12 \mathrm{~A}$ & 120.2 \\
\hline
\end{tabular}




\begin{tabular}{|c|c|c|c|}
\hline $\mathrm{C} 3-\mathrm{C} 4-\mathrm{H} 4 \mathrm{~A}$ & 120.5 & $\mathrm{C} 12-\mathrm{C} 13-\mathrm{C} 14$ & $119.45(18)$ \\
\hline $\mathrm{C} 5-\mathrm{C} 4-\mathrm{H} 4 \mathrm{~A}$ & 120.5 & $\mathrm{C} 12-\mathrm{C} 13-\mathrm{H} 13 \mathrm{~A}$ & 120.3 \\
\hline $\mathrm{C} 4-\mathrm{C} 5-\mathrm{C} 6$ & $121.16(16)$ & $\mathrm{C} 14-\mathrm{C} 13-\mathrm{H} 13 \mathrm{~A}$ & 120.3 \\
\hline $\mathrm{C} 4-\mathrm{C} 5-\mathrm{H} 5 \mathrm{~A}$ & 119.4 & $\mathrm{C} 15-\mathrm{C} 14-\mathrm{C} 13$ & $117.6(2)$ \\
\hline $\mathrm{C} 6-\mathrm{C} 5-\mathrm{H} 5 \mathrm{~A}$ & 119.4 & $\mathrm{C} 15-\mathrm{C} 14-\mathrm{H} 14 \mathrm{~A}$ & 121.2 \\
\hline $\mathrm{C} 5-\mathrm{C} 6-\mathrm{C} 1$ & $120.41(14)$ & $\mathrm{C} 13-\mathrm{C} 14-\mathrm{H} 14 \mathrm{~A}$ & 121.2 \\
\hline $\mathrm{C} 5-\mathrm{C} 6-\mathrm{C} 7$ & $118.19(13)$ & $\mathrm{N} 3-\mathrm{C} 15-\mathrm{C} 14$ & $123.9(2)$ \\
\hline $\mathrm{C} 1-\mathrm{C} 6-\mathrm{C} 7$ & $121.25(13)$ & $\mathrm{N} 3-\mathrm{C} 15-\mathrm{H} 15 \mathrm{~A}$ & 118.0 \\
\hline $\mathrm{O} 1-\mathrm{C} 7-\mathrm{N} 1$ & $123.59(13)$ & $\mathrm{C} 14-\mathrm{C} 15-\mathrm{H} 15 \mathrm{~A}$ & 118.0 \\
\hline $\mathrm{O} 1-\mathrm{C} 7-\mathrm{C} 6$ & $121.89(12)$ & $\mathrm{C} 1-\mathrm{C} 16-\mathrm{H} 16 \mathrm{~A}$ & 109.5 \\
\hline $\mathrm{N} 1-\mathrm{C} 7-\mathrm{C} 6$ & $114.47(11)$ & $\mathrm{C} 1-\mathrm{C} 16-\mathrm{H} 16 \mathrm{~B}$ & 109.5 \\
\hline $\mathrm{N} 2-\mathrm{C} 8-\mathrm{N} 1$ & $117.23(12)$ & $\mathrm{H} 16 \mathrm{~A}-\mathrm{C} 16-\mathrm{H} 16 \mathrm{~B}$ & 109.5 \\
\hline $\mathrm{N} 2-\mathrm{C} 8-\mathrm{S} 1$ & $124.66(11)$ & $\mathrm{C} 1-\mathrm{C} 16-\mathrm{H} 16 \mathrm{C}$ & 109.5 \\
\hline $\mathrm{N} 1-\mathrm{C} 8-\mathrm{S} 1$ & $118.08(10)$ & $\mathrm{H} 16 \mathrm{~A}-\mathrm{C} 16-\mathrm{H} 16 \mathrm{C}$ & 109.5 \\
\hline $\mathrm{N} 2-\mathrm{C} 9-\mathrm{C} 10$ & $113.56(12)$ & $\mathrm{H} 16 \mathrm{~B}-\mathrm{C} 16-\mathrm{H} 16 \mathrm{C}$ & 109.5 \\
\hline $\mathrm{C} 6-\mathrm{C} 1-\mathrm{C} 2-\mathrm{C} 3$ & $0.1(3)$ & $\mathrm{C} 9-\mathrm{N} 2-\mathrm{C} 8-\mathrm{N} 1$ & $173.37(12)$ \\
\hline $\mathrm{C} 16-\mathrm{C} 1-\mathrm{C} 2-\mathrm{C} 3$ & $177.89(19)$ & $\mathrm{C} 9-\mathrm{N} 2-\mathrm{C} 8-\mathrm{S} 1$ & $-4.5(2)$ \\
\hline $\mathrm{C} 1-\mathrm{C} 2-\mathrm{C} 3-\mathrm{C} 4$ & $-0.6(3)$ & $\mathrm{C} 7-\mathrm{N} 1-\mathrm{C} 8-\mathrm{N} 2$ & $-1.7(2)$ \\
\hline $\mathrm{C} 2-\mathrm{C} 3-\mathrm{C} 4-\mathrm{C} 5$ & $0.0(3)$ & $\mathrm{C} 7-\mathrm{N} 1-\mathrm{C} 8-\mathrm{S} 1$ & $176.26(11)$ \\
\hline $\mathrm{C} 3-\mathrm{C} 4-\mathrm{C} 5-\mathrm{C} 6$ & $1.1(3)$ & $\mathrm{C} 8-\mathrm{N} 2-\mathrm{C} 9-\mathrm{C} 10$ & $84.03(18)$ \\
\hline $\mathrm{C} 4-\mathrm{C} 5-\mathrm{C} 6-\mathrm{C} 1$ & $-1.6(2)$ & $\mathrm{N} 2-\mathrm{C} 9-\mathrm{C} 10-\mathrm{C} 11$ & $61.39(18)$ \\
\hline $\mathrm{C} 4-\mathrm{C} 5-\mathrm{C} 6-\mathrm{C} 7$ & $174.02(15)$ & $\mathrm{C} 15-\mathrm{N} 3-\mathrm{C} 11-\mathrm{C} 12$ & $0.8(2)$ \\
\hline $\mathrm{C} 2-\mathrm{C} 1-\mathrm{C} 6-\mathrm{C} 5$ & $0.9(2)$ & $\mathrm{C} 15-\mathrm{N} 3-\mathrm{C} 11-\mathrm{C} 10$ & $-178.50(15)$ \\
\hline $\mathrm{C} 16-\mathrm{C} 1-\mathrm{C} 6-\mathrm{C} 5$ & $-176.76(16)$ & $\mathrm{C} 9-\mathrm{C} 10-\mathrm{C} 11-\mathrm{N} 3$ & $-113.16(15)$ \\
\hline $\mathrm{C} 2-\mathrm{C} 1-\mathrm{C} 6-\mathrm{C} 7$ & $-174.53(14)$ & $\mathrm{C} 9-\mathrm{C} 10-\mathrm{C} 11-\mathrm{C} 12$ & $67.54(19)$ \\
\hline $\mathrm{C} 16-\mathrm{C} 1-\mathrm{C} 6-\mathrm{C} 7$ & $7.8(2)$ & $\mathrm{N} 3-\mathrm{C} 11-\mathrm{C} 12-\mathrm{C} 13$ & $-0.1(3)$ \\
\hline $\mathrm{C} 8-\mathrm{N} 1-\mathrm{C} 7-\mathrm{O} 1$ & $5.5(2)$ & $\mathrm{C} 10-\mathrm{C} 11-\mathrm{C} 12-\mathrm{C} 13$ & $179.20(17)$ \\
\hline $\mathrm{C} 8-\mathrm{N} 1-\mathrm{C} 7-\mathrm{C} 6$ & $-171.85(12)$ & $\mathrm{C} 11-\mathrm{C} 12-\mathrm{C} 13-\mathrm{C} 14$ & $-1.0(3)$ \\
\hline $\mathrm{C} 5-\mathrm{C} 6-\mathrm{C} 7-\mathrm{O} 1$ & $-128.16(16)$ & $\mathrm{C} 12-\mathrm{C} 13-\mathrm{C} 14-\mathrm{C} 15$ & $1.2(3)$ \\
\hline $\mathrm{C} 1-\mathrm{C} 6-\mathrm{C} 7-\mathrm{O} 1$ & $47.4(2)$ & $\mathrm{C} 11-\mathrm{N} 3-\mathrm{C} 15-\mathrm{C} 14$ & $-0.5(3)$ \\
\hline $\mathrm{C} 5-\mathrm{C} 6-\mathrm{C} 7-\mathrm{N} 1$ & $49.22(18)$ & $\mathrm{C} 13-\mathrm{C} 14-\mathrm{C} 15-\mathrm{N} 3$ & $-0.5(4)$ \\
\hline $\mathrm{C} 1-\mathrm{C} 6-\mathrm{C} 7-\mathrm{N} 1$ & $-135.22(14)$ & & \\
\hline
\end{tabular}

Hydrogen-bond geometry $\left(A,{ }^{\circ}\right)$

$\mathrm{Cg} 2$ is the centroid of the $\mathrm{C} 1-\mathrm{C} 6$ ring.

\begin{tabular}{lllll}
\hline$D-\mathrm{H} \cdots A$ & $D-\mathrm{H}$ & $\mathrm{H} \cdots A$ & $D \cdots A$ & $D-\mathrm{H} \cdots A$ \\
\hline $\mathrm{N} 2-\mathrm{H} 1 N 2 \cdots \mathrm{O} 1$ & $0.842(17)$ & $1.971(18)$ & $2.6550(16)$ & $137.8(16)$ \\
$\mathrm{N} 1-\mathrm{H} 1 N 1 \cdots \mathrm{N} 3^{\mathrm{i}}$ & $0.875(17)$ & $2.061(17)$ & $2.9366(17)$ & $180(3)$ \\
$\mathrm{C} 16-\mathrm{H} 16 A \cdots \mathrm{S} 1^{\mathrm{i}}$ & 0.96 & 2.86 & $3.782(2)$ & 162 \\
$\mathrm{C} 12-\mathrm{H} 12 A \cdots C g 2^{\mathrm{ii}}$ & 0.93 & 2.92 & $3.7724(19)$ & 153 \\
\hline
\end{tabular}

Symmetry codes: (i) $-x+1,-y+2,-z$; (ii) $-x+1,-y+1,-z$. 